\title{
Robust recognition technique for handwritten Kannada character recognition using capsule networks
}

\author{
N. Shobha Rani, Manohar N., Hariprasad M., Pushpa B. R. \\ Department of Computer Science, Amrita School of Arts and Sciences, Mysuru Campus, Amrita Vishwa Vidyapeetham, India
}

\begin{tabular}{l} 
Article Info \\
\hline Article history: \\
Received Apr 16, 2021 \\
Revised Jun 16, 2021 \\
Accepted Jul 11, 2021 \\
\hline Keywords: \\
Capsule networks \\
CNN \\
Document images \\
Handwritten Kannada \\
characters \\
Image processing \\
OCR
\end{tabular}

\begin{abstract}
Automated reading of handwritten Kannada documents is highly challenging due to the presence of vowels, consonants and its modifiers. The variable nature of handwriting styles aggravates the complexity of machine based reading of handwritten vowels and consonants. In this paper, our investigation is inclined towards design of a deep convolution network with capsule and routing layers to efficiently recognize Kannada handwritten characters. Capsule network architecture is built of an input layer, two convolution layers, primary capsule, routing capsule layers followed by trilevel dense convolution layer and an output layer. For experimentation, datasets are collected from more than 100 users for creation of training data samples of about 7769 comprising of 49 classes. Test samples of all the 49 classes are again collected separately from 3 to 5 users creating a total of 245 samples for novel patterns. It is inferred from performance evaluation; a loss of $0.66 \%$ is obtained in the classification process and for 43 classes precision of $100 \%$ is achieved with an accuracy of $99 \%$. An average accuracy of $95 \%$ is achieved for all remaining 6 classes with an average precision of $89 \%$.
\end{abstract}

This is an open access article under the CC BY-SA license.

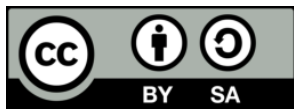

\section{Corresponding Author:}

N. Shobha Rani

Department of Computer Science, Amrita School of Arts and Sciences, Mysuru Campus

Amrita Vishwa Vidyapeetham, India

Email: n_shobharani@my.amrita.edu

\section{INTRODUCTION}

Recognition of hand written characters from document images is an open research problem in the area of optical character recognition (OCR). Realization of higher accuracy is quite demanding especially for hand written documents. In this connection recognition of South Indian languages is challenging compared to North Indian hand written documents. In particular, a research exploration towards Kannada handwritten characters is highly difficult due to its large number of character classes and complex geometrical topography [1]. Kannada character sets for recognition is typically composed of 13 vowels and 25 consonants as shown in Figure 1.

In addition to the basic Kannada character sets, vowel conjuncts of 13 and consonants conjuncts of 25 are present as shown in Figure 2. The complexity of character recognition is dependent on the character set and its number of classes. In Kannada number of character classes will be $13 * 25 * 25$ [2]. Increasing in number of classes aggravates the challenges in feature analysis and classification process. Additionally, challenges inherent in hand written datasets introduce barriers to attain higher recognition accuracy.

Ample research investigations were reported earlier for classification of hand written Kannada characters. The techniques of character classification are based on machine learning and deep learning procedures [3], [4]. Although machine learning based techniques perform better towards classification of Kannada character classes, the results are appreciable only for limited number of classes. Thus, the research 
efforts towards classification of large number of character sets are accomplished through deep learning procedures. OCR technologies are generally developed addressing the script based recognition needs. OCR's that perform better towards one script results in poor performance towards other scripts. The performance of OCR also depends on printed/hand written datasets, number of writing styles, script type and document layout. Some of the OCR systems are template based that follow strict rules during input image acquisition stage. Several recent studies in [5]-[10] proposed hand written character classification using deep learning based models.

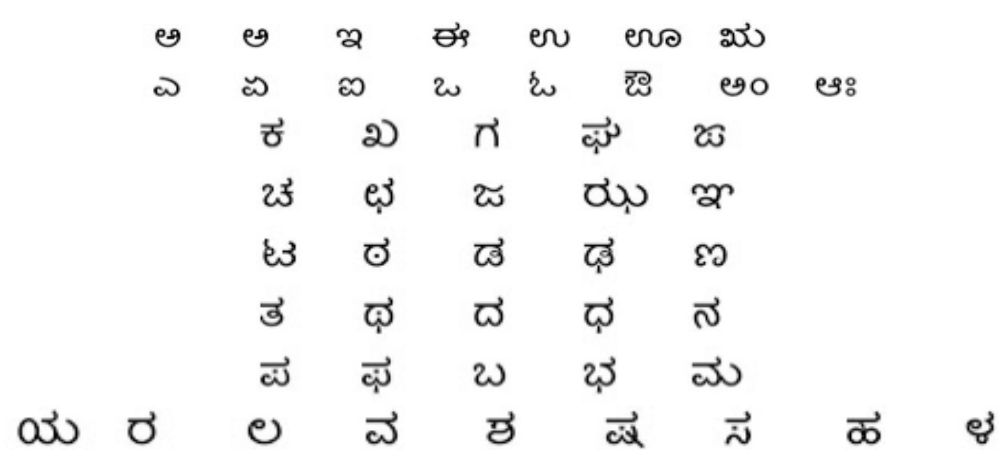

Figure 1. Kannada character set

\begin{tabular}{|c|c|c|c|}
\hline అ & ఆ & 2 & ఈs \\
\hline eN & eת & య & $\omega$ \\
\hline$\omega$ & $\varpi$ & ఒ & \{ \\
\hline ఔ & & & \\
\hline
\end{tabular}

\begin{tabular}{|c|c|c|c|c|c|}
\hline 항 & 2 & $\vec{n}$ & $\vec{\varphi}$ & $\varkappa$ & ఒ3 \\
\hline ๔ & ఒ & ఝు & হ & $\varepsilon 3$ & ఠ \\
\hline$\vec{\omega}$ & $\vec{\varphi}$ & ణ & उ & థ & $\vec{ద}$ \\
\hline ధ & $\vec{\sim}$ & $\vec{\omega}$ & $\ddot{\varphi}$ & బ & భ \\
\hline డు & యో & $\delta$ & $\omega$ & $e$ & $\vec{\omega}$ \\
\hline ர & $\overrightarrow{c \vec{\omega}}$ & $\vec{\sim}$ & $\varpi$ & 83 & $\omega$ \\
\hline
\end{tabular}

Figure 2. Kannada character set vowels and consonants conjuncts

In the literature there exists more number of research attempts in the area of Kannada hand written character recognition. Some of the significant research include Joe et al. [11] proposed offline character recognition system for hand written characters using convolutional neural network. It is proved that hand crafted features are not required for hand written character classification. In an another work, Ramesh et al. [12] also employed convolutional neural network for handwritten Kannada character recognition. An accuracy of 93.2\% is obtained towards classification of basic vowels and consonants. Further Sandhya et al. [13] investigated a framework for degraded Kannada character recognition by addressing character stroke breakages, complex compound characters in printed documents using machine learning based techniques. In an another work Ucar et al. [14] used capsule network for classification of Kannada handwritten digit datasets. A comparison is performed using convolutional neural networks and observed that Capsule Network model provide better efficiency.

Mahapatra et al. [15] applied learning viz auto encoders to classify handwritten Kannada characters using KNN and SVM classifiers. Later, capsule networks for recognition of low resource languages such as Kannada is investigated by Abeysinghe and Perera [16]. The applicability of the model is extended to languages such as oriya and Sinhala character sets. In a different work Ramesh and Kumar [17] applied convolutional neural network for recognition of Kannada handwritten words, SVM classifier is employed as part of fully connected layer. Mamatha [18] proposed a dataset for handwritten Kannada vowels and classified the local binary pattern, run length count and chain codes using K-means clustering. Mahapathra et al. [19] proposed a generator based methods for offline handritten character recognition using convolutional auto encoder with GAN architecture. Indira and Selvi [20] reviewed various methods for Kannada printed character recognition. In another work applicability of wavelet transform and structural features are adapted for Kannada handwritten character recognition by Pasha and Padma [21]. The experimentations were conducted on hand written numerals. 
Recognition of Arabic characters is proposed by Ahamad et al. [22] using convolutional neural networks trained using different learning rates. In a subsequent work, Boufenar et al. [23] classified handwritten Arabic characters using convolutional networks through transfer learning approach. Followed by this Vaidya et al. [24] carried out handwritten character classification using deep learning convolutional model. Experimentations are conducted on handwritten digits. A character recognition model is proposed by Ram et al. [25] using deep convolutional neural network. In another work Wick et al. [26] applied deep learning packages for optical character recognition. A series of attempts by Zhao et al. [27], Selmi et al. [28], Iamsa et al. [29], Nair et al. [30], Alif et al. [31] used variety of deep learning architectures for various handwritten character recognition problems.

From the literature, it is evident that deep learning systems are widely used for recognition of handwritten characters. However the focus towards classification of South Indian scripts is rarely noticed. Though the deep learning architectures proposed for character and digit classification are successful, the realization of high accuracies with respect to handwritten character recognition is a failure. In a couple of works satisfactory progress is achieved towards North Indian scripts based on Devanagari. Thus, there exists a scope for applying deep learning based models for handwritten Kannada character recognition. In the proposed work, capsule network based deep learning model is applied to classify Kannada handwritten characters.

\section{PROPOSED METHOD}

Representation of geometrical relationships via artificial neural networks is the inclination of capsule networks $(\mathrm{CN})$. It is a type of convolutional neural network that provides a way of reusing the output produced in a specific iteration to stabilize the outputs to be obtained in subsequent iterations. CN's possess strange abilities in interpretation of feature maps to various images, this will help in various image classification and recognition tasks. The features employed for learning are present at the core of convolution layers in CN's. With regard to handwritten character recognition problem, learning from the level of pixels with respect to edges and color suffices the image recognition tasks. Unlike convolutional neural network $(\mathrm{CNN})$, the CN's are not adaptable to the change in small information in the parts of image. Therefore in the proposed method an attempt is made towards classification of handwritten Kannada characters using CN's. Figure 3 depicts the architecture of capsule network to perform the classification of handwritten Kannada characters. Architecture of $\mathrm{CN}$ is comprised of an input layer followed by two levels of convolution layers, two levels of capsule layers preceded by squash and squeeze functions and a multilevel upsampling followed by an output layer.

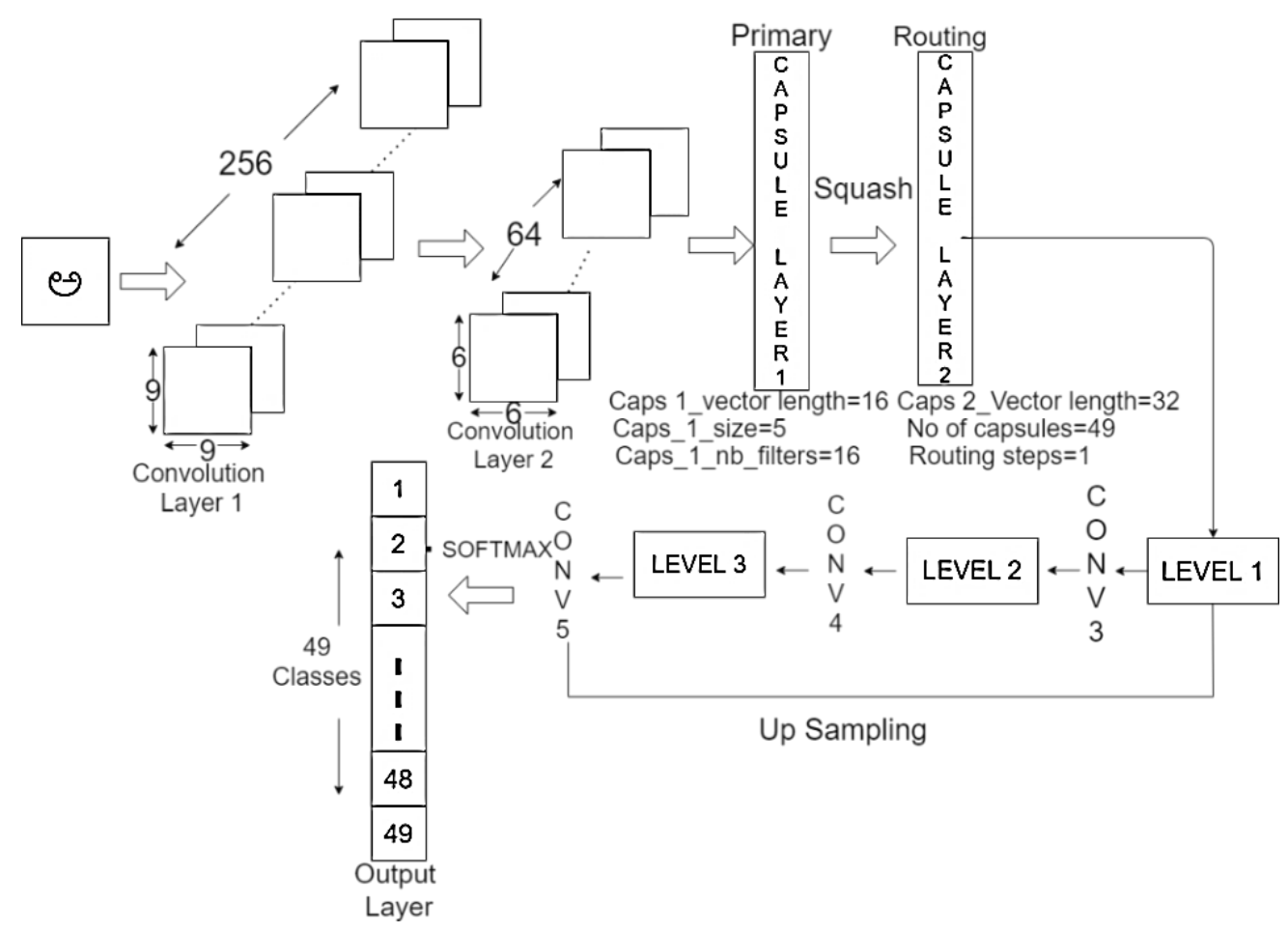

Figure 3. Architecture diagram of capsule network 
Input layer is built of $\mathrm{m}^{*} \mathrm{n}$ number of neurons for an image of dimension $\mathrm{m}^{*} \mathrm{n}$ after which convolution layers $C_{1}$ and $C_{2}$. $C_{1}$ built of 256 convolution kernels of dimensions $9 * 9$ and $C 2$ further down samples input from $\mathrm{C}_{1}$. Convolution layer $\mathrm{C}_{2}$ is composed of 64 convolution kernels each of dimension $6 * 6$. Subsequently the down sampled outcomes produced from convolution layer $\mathrm{C}_{2}$ are subject to capsule layer in two levels. In $\mathrm{CNN}$ each node in the input layer represents a pixel, input data from image is transformed to the subsequent layers for down sampling. In the down sampling process their exist a loss of positional attributes that results in loss of location of object structures and direction and its gradient directions. Therefore, capsule layers are introduced in the proposed architecture that can retain more information about object structures in terms of its position, size and orientation. Thus the information prevalent to perform Kannada hand written character classification can be stored in the form of capsules. Capsules are vectors that emphasize the positional attributes of objects along with spatial invariance information. Each capsule carries a degree of features across a range of spatial variations adequate to handle the handwritten Kannada character recognition task. Hence the information in a particular capsule helps in better identification of a character. In proposed architecture, a capsule vector of length 16 is employed in the layer 1 with each capsule size of 5 and number of filters as 16.

In capsule layer 2, capsule vector length of 32 with number of capsules 49 and a routing step size of one is adapted. The length of capsule vector represents the probability of existence of an object with its orientation details and it also acts as one of the instantiation parameters. Activation of capsules at layer 1 assists in making predictions and also instantiation of parameters in the subsequent capsule layers. In level one, 16 nodes are used in each capsule that represents 16 different feature dimensions for one character. The output of a capsule layer one is a vector comprising of properties such as width, scale, stroke thickness and square of a particular character. The elements of the capsule vector are delimited between the intervals of 0-1 indicating the probability of affinity to each class. Whenever multiple predictions from two capsule layers commensurate that results in activation of a neurons in capsules in the higher levels. Additionally these activated neurons from the capsule are subject to squash and squeeze functions as follows. Squash and squeeze are activation functions which are applied over the outputs produced by the capsule layers [32]. These functions are mainly useful in inferring nonlinear relationships within the data and typically act as activation layers by returning a vector of elements that falls in the range of 0 and 1 . Squash is a special activation function that carries out the normalization on the scaled output values produced by capsule layers. If $V e c_{j}$ represents the output vector returned by squash function, $j$ represents a capsule in the layer $l$, $S_{j}$ represents a vector of scalars produced by capsule $j$ in layer $l$ then activation obtained through squash function is given by (1).

$$
V e c_{j}=\frac{\left\|s c a_{j}\right\|^{2}}{1+\left\|s c a_{j}\right\|^{2}} x \frac{s_{j}}{\left\|s_{j}\right\|}
$$

Thus $V e c_{j}$ is a normalized vector returned by applying squash function on $s c a_{j}$. Further $V e c_{j}$ represents the route through which data from various capsules to be trained subsequently.

Figure 4 gives the illustration of data being routed after applying squash function. The outputs obtained from routing capsule layers are further subject to excitation using squeeze function. Channel wise feature map vectors are recalibrated using squeeze by explicitly modeling inter-dependencies between the features. This will increase the accuracy via better representation of channel wise features for classification. Following the squeeze excitation there are repeated convolutions at level 1, level 2, and level 3 through convolution layer 3, convolution layer 4 and convolution layer 5. Additionally a softmax activation function is applied on the up sampled output vectors produced from up convolution process. Softmax function helps in mapping of the output vectors to desired number of classes.

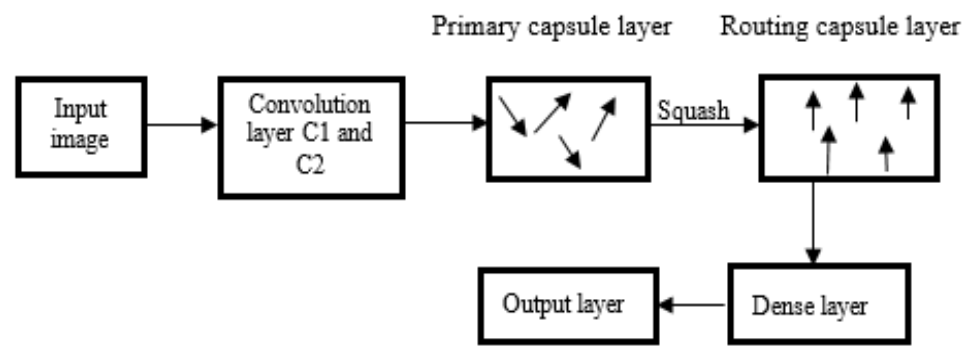

Figure 4. Routing of data-capsule layers using SQUASH 


\section{EXPERIMENTATION}

In the proposed work, performance of CN's is tested with 7,769 training datasets, 245 testing datasets and 490 samples for validation. A training and validation datasets are the samples of datasets that are held back for learning and unbiased performance evaluation. Test datasets are collected separately from about 200 users with references of 1-2 samples for each of the 49 classes resulting into 245 samples. To estimate the performance of trained model, validation is conducted with 490 samples for 49 classes with 10samples in each class. Validation data is mainly used to understand the accuracy estimate of the CN. Table 1 shows the details of datasets used for experimentation for all the 49 classes.

Table 1. Dataset details of Kannada handwritten character recognition

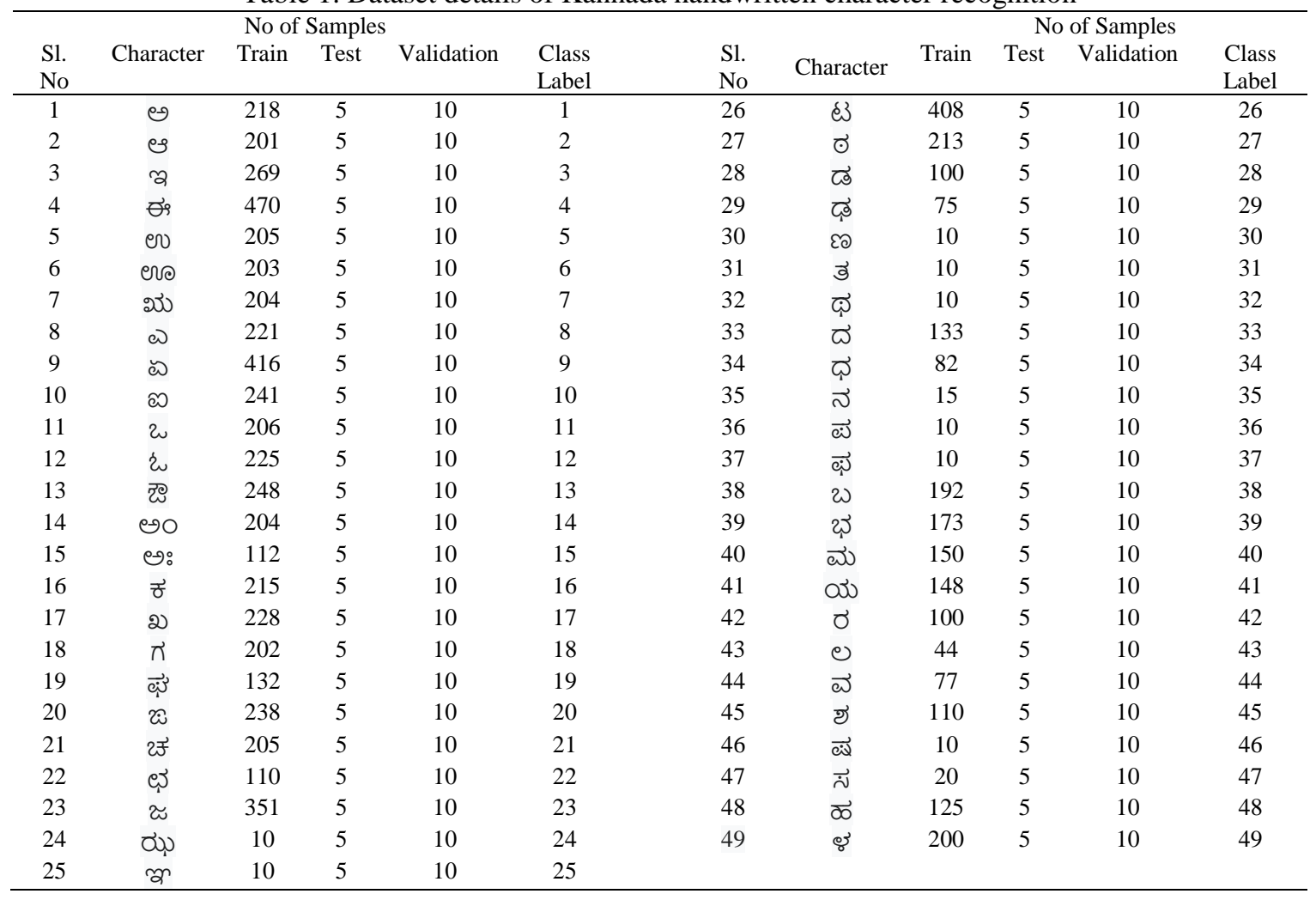

A mini batch gradient descent approach with batch size of 50 and number of training steps equal to 7000 is considered for evaluation. Learning frameworks used includes TensorFlow, Keras 2.1.5 and OpenCV 4.5.1 and the primary development environment used is Spyder. The computing resources employed for simulation includes ASUS laptop with 16 GB RAM, Intel core I7 processor and an additional GPU memory of $4 \mathrm{~GB}$. Each image sample considered for experimentation is a gray scale image with $28 * 28$ pixels. The model is tested with different hyper parameters by modifying the batch sizes from 10-50. Samples of training, validation and test datasets are randomly chosen and few samples are shown in Figure 5.
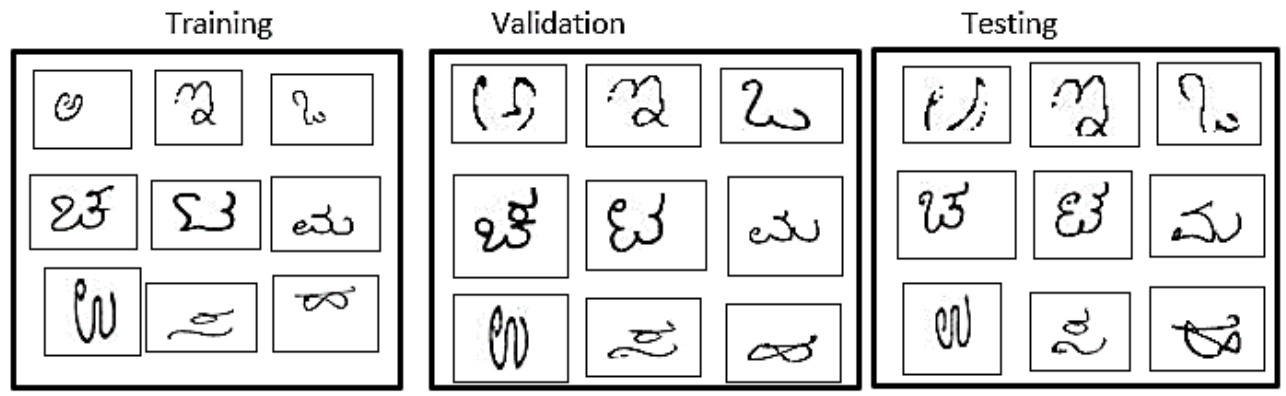

Figure 5. Sample instances from training validation and testing 
The margin loss obtained after instantiation of vectors from routing capsules with respect to the trained data is shown in the Figure 6. About 10-12 hours of time is consumed for training the CN with 7,769 samples of 49 classes and routing step size of 0.01 . Reconstruction loss obtained for 7000 training steps after routing process via primary and routing capsules is shown in Figure 7.

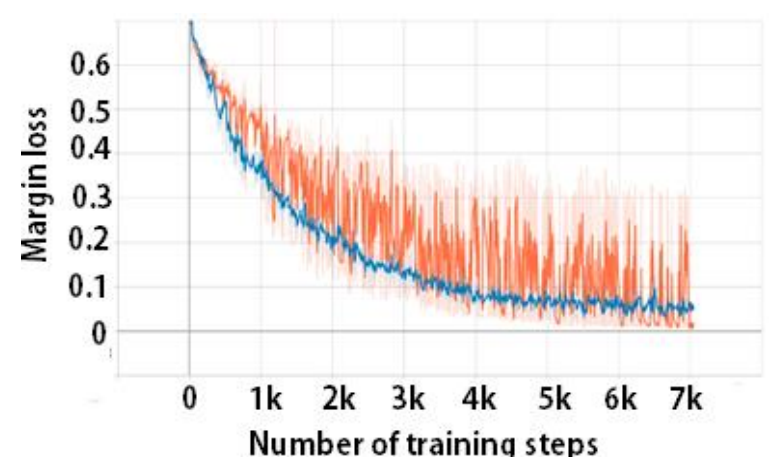

Figure 6. Margin loss-training and validation samples

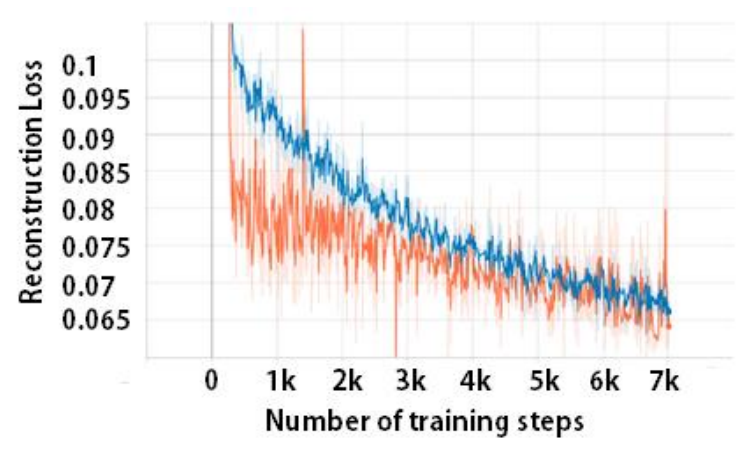

Figure 7. A reconstruction loss-training and validation samples

It is observed that a loss of 0.66 is found at the end of 7000 training steps with the validation dataset samples and total loss for all the 49 character classes from the capsule network is found to be less than $3 \%$. If ' $M$ ' represents reconstruction loss, ' $T$ ' as total loss and ' $\beta$ ' represents as scaling factor than total loss ' $T$ ' is given by (2). Figure 8 shows the total loss obtained during classification.

$$
T=M+\beta(R)
$$

The overall accuracy obtained is found to be $97 \%$ with average accuracy of $95 \%$ across all classes. Figure 9 depicts the accuracy obtained towards classification of hand written Kannada characters. Table 2 shows the performance metrics in terms of precision, recall and accuracy with respect to every class. From the Table 2, it is evident that 48 out of 49 classes are classified with an average accuracy of more than $91.5 \%$. i.e. 4 out of 5 samples are classified on an average for 18 classes and 5 out of 5 samples are correctly classified for all the remaining classes. Experimentation on the proposed datasets are also conducted using Inception V3 network and accuracy of the handwritten Kannada character recognition is found to be $95 \%$. Cross entropy is also evaluated to depict the distance between the predicted and actual class labels. Cross entropy depicts the loss occurred towards Kannada handwritten character recognition. Figures 10 and 11 depicts the performance of inception V3 network in terms of accuracy v/s number of iterations and cross entropy.

It is evident from the experimentation that performance of $\mathrm{CN}$ architecture is much better compared to inception V3 network. Also the loss occurred with regard to $\mathrm{CN}$ is much less compared to inception V3. Moreover, $\mathrm{CN}$ helps in reduced loss of features from the image during the convolution process. Thus, robustness of $\mathrm{CN}$ is much higher than the inception $\mathrm{V} 3$ network.

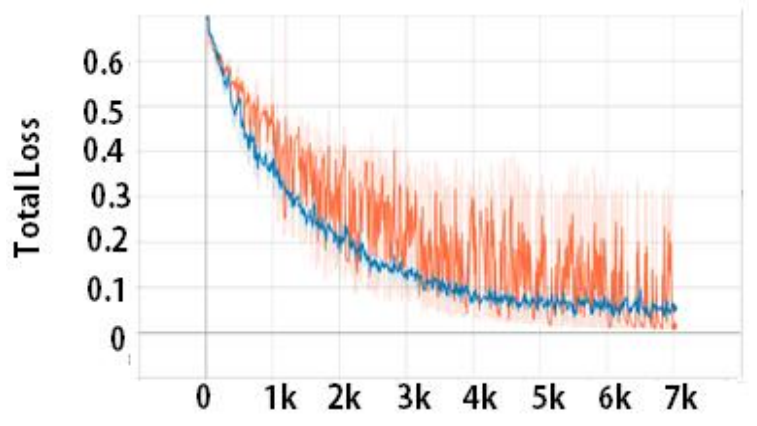

Figure 8. Total loss training and validation samples

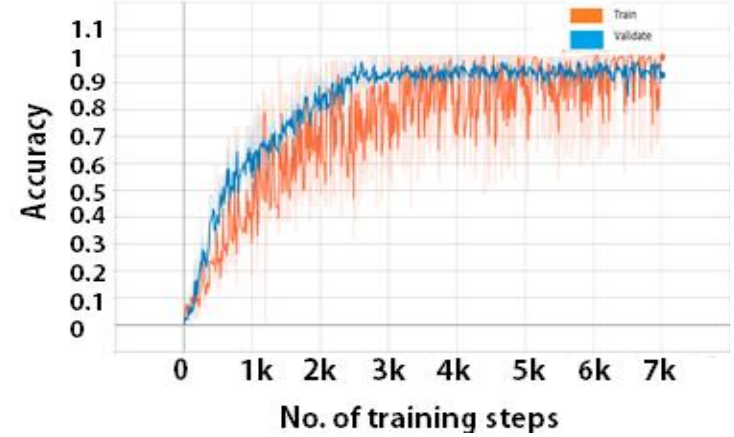

Figure 9. Accuracy-training vs validation samples 
Table 2. Performance metrics of capsule networks with test datasets

\begin{tabular}{crrrrrrr}
\hline Class label & Precision & Recall & F1-Score & Class label & Precision & Recall & F1-Score \\
\hline 1 & 1.00 & 1.00 & 1.00 & 26 & 1.00 & 1.00 & 1.00 \\
2 & 1.00 & 1.00 & 1.00 & 27 & 0.95 & 1.00 & 0.97 \\
3 & 1.00 & 1.00 & 1.00 & 28 & 0.77 & 1.00 & 0.87 \\
4 & 1.00 & 1.00 & 1.00 & 29 & 1.00 & 1.00 & 1.00 \\
5 & 1.00 & 1.00 & 1.00 & 30 & 0.90 & 1.00 & 0.95 \\
6 & 1.00 & 1.00 & 1.00 & 31 & 1.00 & 1.00 & 1.00 \\
7 & 1.00 & 1.00 & 1.00 & 32 & 0.96 & 1.00 & 0.98 \\
8 & 1.00 & 1.00 & 1.00 & 33 & 0.92 & 1.00 & 0.96 \\
9 & 1.00 & 1.00 & 1.00 & 34 & 0.83 & 1.00 & 0.91 \\
10 & 0.99 & 1.00 & 1.00 & 35 & 1.00 & 1.00 & 1.00 \\
11 & 1.00 & 1.00 & 1.00 & 36 & 0.80 & 0.80 & 0.80 \\
12 & 1.00 & 1.00 & 1.00 & 37 & 1.00 & 1.00 & 1.00 \\
13 & 1.00 & 1.00 & 1.00 & 38 & 1.00 & 0.80 & 0.89 \\
14 & 1.00 & 1.00 & 1.00 & 39 & 1.00 & 0.80 & 0.89 \\
15 & 0.93 & 1.00 & 0.97 & 40 & 0.00 & 0.00 & 0.00 \\
16 & 1.00 & 1.00 & 1.00 & 41 & 1.00 & 1.00 & 1.00 \\
17 & 0.91 & 1.00 & 0.95 & 42 & 1.00 & 1.00 & 1.00 \\
18 & 1.00 & 1.00 & 1.00 & 43 & 0.75 & 0.60 & 0.67 \\
19 & 1.00 & 1.00 & 1.00 & 44 & 0.86 & 1.00 & 0.92 \\
20 & 1.00 & 1.00 & 1.00 & 45 & 0.94 & 1.00 & 0.97 \\
21 & 1.00 & 1.00 & 1.00 & 46 & 1.00 & 0.80 & 0.89 \\
22 & 1.00 & 0.89 & 0.94 & 47 & 1.00 & 1.00 & 1.00 \\
23 & 1.00 & 1.00 & 1.00 & 48 & 1.00 & 1.00 & 1.00 \\
24 & 1.00 & 1.00 & 1.00 & 49 & 1.00 & 1.00 & 1.00 \\
25 & 1.00 & 1.00 & 1.00 & & & & \\
Overall Accuracy & & & & & & & 0.97 \\
Macro Average & & & & & & & 0.95 \\
Weighted Average & & & & & & & 0.97 \\
\hline
\end{tabular}

accuracy_1

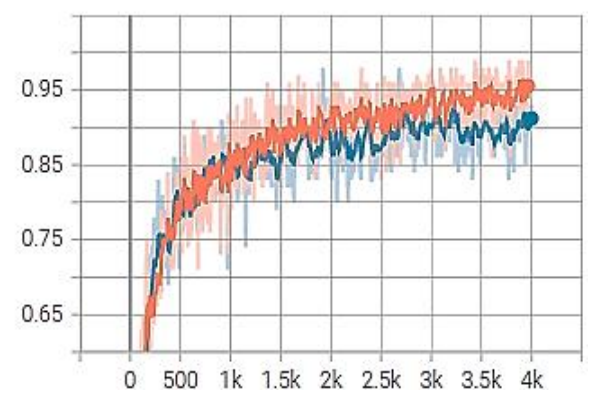

Figure 10. Accuracy of the inception V3 network

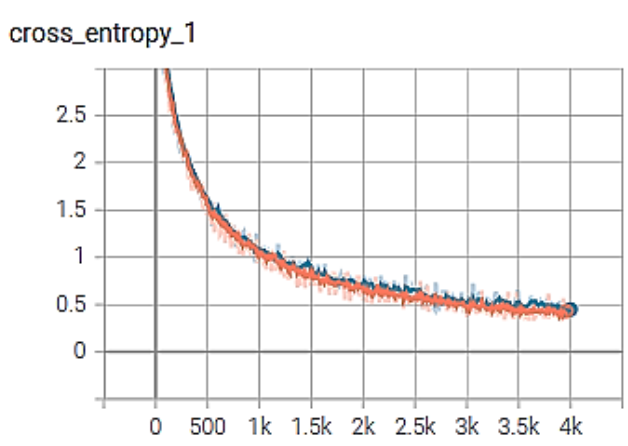

Figure 11. Cross entropy of inception V3 network

\section{CONCLUSION}

Deep learning networks are used extensively in the recent days to deal with variety of pattern recognition problems. In this work, we have investigated the applicability of $\mathrm{CN}$ towards classification of handwritten Kannada characters. A design of $\mathrm{CN}$ architecture is proposed with tunable hyper parameters. Comparison of proposed model is carried out with inception V3 model and proved that an accuracy of $99 \%$ is achieved with $\mathrm{CN}$ model. In future, the model can be extended to classify simple compound and multi compound characters with increased number of data samples.

\section{ACKNOWLEDGEMENTS}

We honestly acknowledge our Chancellor, Mata Amritanandamayi Devi for giving us an opportunity to carry out the research at Amrita Vishwa Vidyapeetham, Mysuru Campus. Also, we are grateful for the facilitation of hardware and software resources at School of Arts and Sciences, Mysuru Campus.

\section{REFERENCES}

[1] S. Bag and G. Harit, "A survey on optical character recognition for bangla and devanagari scripts," Sadhana, vol. 38, no. 1, pp. 133-168, 2013, doi: 10.1007/s12046-013-0121-9.

[2] G. S. G. Rajput and R. Horakeri, "Zone based handwritten Kannada character recognition using crack code and SVM," in 2013 International Conference on Advances in Computing, Communications and Informatics (ICACCI), 2013, pp. 1817-1821, doi: 10.1109/ICACCI.2013.6637457. 
[3] A. Pal and H. Singh, "Handwritten english character recognition using neural network," International Journal of Computer Science and Communication (IJCSC), vol. 1, no. 2, pp. 141-144, 2010.

[4] N. S. Rani, T. Vasudev, M. Chandrajith, and N. Manohar, "2D morphable feature space for handwritten character recognition," Procedia Computer Science, vol. 167, pp. 2276-2285, 2020.

[5] S. Acharya, A. K. Pant, and P. K. Gyawali, "Deep learning based large scale handwritten Devanagari character recognition," 2015 9th International conference on software, knowledge, information management and applications (SKIMA), 2015, pp. 1-6, doi: 10.1109/SKIMA.2015.7400041.

[6] S. Roy, N. Das, M. Kundu, and N. Nasipuri, "Handwritten isolated bangla compound character recognition: A new benchmark using a novel deep learning approach," Pattern Recognition Letters, vol. 90, pp. 15-21, 2017, doi: 10.1016/j.patrec.2017.03.004.

[7] L. Meng, C. V. Aravinda, K. U. K. Reddy, T. Izumi, and K. Yamazaki, "Ancient asian character recognition for literature preservation and understanding," Euro-Mediterranean Conference, vol. 11196, 2018, pp. 741-751, doi: 10.1007/978-3-03001762-0_66.

[8] N. S. Rani, N. Chandan, A. S. Jain, and H. R. Kiran, "Deformed character recognition using convolutional neural networks," International Journal of Engineering and Technology, vol. 7, no. 3, pp. 1599-1604, 2018, doi: 10.14419/ijet.v7i3.14053.

[9] N. S. Rani, A. C. Subramani, A. Kumar, and B. R. Pushpa, "Deep learning network architecture based kannada handwritten character recognition," 2020 Second International Conference on Inventive Research in Computing Applications (ICIRCA), 2020, pp. 213-220, doi: 10.1109/ICIRCA48905.2020.9183160.

[10] M. A. Pragathi, K. Priyadarshini, S. Saveetha, A. S. Banu, and K. M. Aarif, "Handwritten tamil character recognition using deep learning," 2019 International Conference on Vision Towards Emerging Trends in Communication and Networking (ViTECoN), 2019, pp. 1-5, doi: 10.1109/ViTECoN.2019.8899614.

[11] K. G. Joe, M. Savit, and K. Chandrasekaran, "Offline character recognition on segmented handwritten kannada characters," 2019 Global Conference for Advancement in Technology (GCAT), 2019, pp. 1-5, doi: 10.1109/GCAT47503.2019.8978320.

[12] G. Ramesh, G. N. Sharma, J. M. Balaji, and H. N. Champa, "Offline Kannada handwritten character recognition using convolutional neural networks," 2019 IEEE International WIE Conference on Electrical and Computer Engineering (WIECONECE), 2019, pp. 1-5, doi: 10.1109/WIECON-ECE48653.2019.9019914.

[13] N. Sandhya, R. Krishnan, and D. R. Babu, "A framework for degraded Kannada character recognition," International Conference on Image Processing and Capsule Networks, vol. 1200, 2020, pp. 735-745, doi: 10.1007/978-3-030-51859-2_67.

[14] E. Uçar, and M. Uçar, "Applying capsule network on Kannada-MNIST handwritten digit dataset," Natural and Engineering Sciences, vol. 4, no. 3, pp. 100-106, 2019.

[15] D. Mahapatra, C. Choudhury, and R. K. Karsh, "Handwritten character recognition using KNN and SVM based classifier over feature vector from autoencoder," International Conference on Machine Learning, Image Processing, Network Security and Data Sciences, vol. 1240, 2020, pp. 304-317, doi: 10.1007/978-981-15-6315-7_25.

[16] C. Abeysinghe, I. Perera, and D. A. Meedeniya, "Capsule networks for character recognition in low resource languages," Machine Vision Inspection Systems, vol. 2, pp. 23-46, 2021, doi: 10.1002/9781119786122.ch2.

[17] G. Ramesh and S. Kumar, "Recognition of Kannada handwritten words using SVM classifier with convolutional neural network," 2020 IEEE Region 10 Symposium (TENSYMP), 2020, pp. 1114-1117, doi: 10.1109/TENSYMP50017.2020.9231003.

[18] H. R. Mamatha, "Dataset building for handwritten Kannada vowels using unsupervised and supervised learning methods," International Symposium on Signal Processing and Intelligent Recognition Systems-SIRS 2020, Chennai, India, vol. 1365, 2021, pp. 75-89, doi: 10.1007/978-981-16-0425-6_6.

[19] D. Mahapatra, C. Choudhury, and R. K. Karsh, "Generator based methods for off-line handwritten character recognition," 2020 Advanced Communication Technologies and Signal Processing (ACTS), 2020, pp. 1-6, doi: 10.1109/ACTS49415.2020.9350491.

[20] K. Indira and S. S. Selvi, "Kannada character recognition system a review," arXiv preprint arXiv:1001.5352, 2010.

[21] S. Pasha and M. C. Padma, "Handwritten Kannada character recognition using wavelet transform and structural features," 2015 International Conference on Emerging Research in Electronics, Computer Science and Technology (ICERECT), 2015, pp. 346-351, doi: 10.1109/ERECT.2015.7499039.

[22] S. B. Ahmed, S. Naz, M. I. Razzak, and R. Yousaf, "Deep learning based isolated arabic scene character recognition," 2017 1st International Workshop on Arabic Script Analysis and Recognition (ASAR), 2017, pp. 46-51, doi: 10.1109/ASAR.2017.8067758.

[23] C. Boufenar, A. Kerboua, and M. Batouche, "Investigation on deep learning for off-line handwritten Arabic character recognition,” Cognitive Systems Research, vol. 50, pp. 180-195, 2018, doi: 10.1016/j.cogsys.2017.11.002.

[24] R. Vaidya, D. Trivedi, S. Satra, and M. Pimpale, "Handwritten character recognition using deep-learning," 2018 Second International Conference on Inventive Communication and Computational Technologies (ICICCT), 2018, pp. 772-775, doi: 10.1109/ICICCT.2018.8473291.

[25] S. Ram, S. Gupta, and B. Agarwal, "Devanagri character recognition model using deep convolution neural network," Journal of Statistics and Management Systems, vol. 21, no. 4, pp. 593-599, 2018, doi: 10.1080/09720510.2018.1471264.

[26] C. Wick, C. Reul, and F. Puppe, "Calamari-a high-performance tensorflow-based deep learning package for optical character recognition," Digital Humanities Quarterly, vol. 14, no. 2, pp. 1-12, 2020.

[27] Z. Zhao, M. Jiang, S. Guo, Z. Wang, F. Chao, and K. C. Tan, "Improving deep learning based optical character recognition via neural architecture search," 2020 IEEE Congress on Evolutionary Computation (CEC), 2020, pp. 1-7, doi: 10.1109/CEC48606.2020.9185798.

[28] Z. Selmi, M. B. Halima, and A. M. Alimi, "Deep learning system for automatic license plate detection and recognition," 2017 14th IAPR international conference on document analysis and recognition (ICDAR), vol. 1, 2017, pp. 1132-1138, doi: 10.1109/ICDAR.2017.187.

[29] S. Iamsa-at and P. Horata, "Handwritten character recognition using histograms of oriented gradient features in deep learning of artificial neural network," 2013 international conference on IT convergence and security (ICITCS), 2013, pp. 1-5, doi: 10.1109/ICITCS.2013.6717840.

[30] B. B. Nair, C. A. Krishnan, J. Johns, B. S. Jain, and B. Sarath, "An enhanced approach for binarizing and segmenting degraded ayurvedic medical prescription," International Journal of Pharmaceutical Research, vol. 12, no. 3, pp. 783-790, 2020, doi: $10.31838 / \mathrm{ijpr} / 2020.12 .03 .112$.

[31] M. A. R. Alif, S. Ahmed and M. A. Hasan, "Isolated bangla handwritten character recognition with convolutional neural network," 2017 20th International conference of computer and information technology (ICCIT), 2017, pp. 1-6, doi: 10.1109/ICCITECHN.2017.8281823.

[32] D. Goswami, "Application of capsule networks for image classification on complex datasets," M.S. thesis, University of Illinois at Urbana-Champaign, US, 2019. 


\section{BIOGRAPHIES OF AUTHORS}

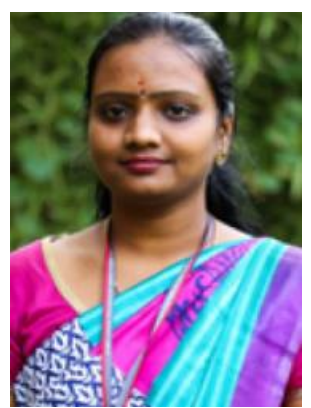

N. Shobha Rani (D) 8d SC P is awarded with Ph.D degree in Computer Science from University of Mysore in the year 2017. She is currently working as Associate Professor in Department of Computer Science, Amrita School of Arts and Sciences, Mysuru Campus. She has published more than $45+$ research articles in reputed international journals and conferences and indexed in SCOPUS/SCI. Her areas of research include document image processing, optical character recognition, pattern classification, machine learning, deep learning and computer vision. She can be contacted at email: n_shobharani@my.amrita.edu

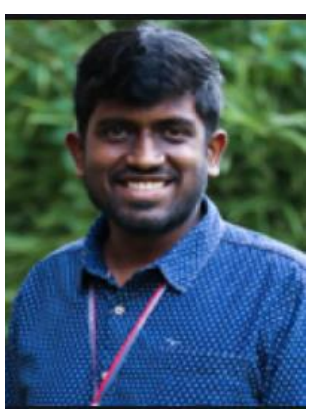

N. Manohar (iD 81 SC P is awarded with Ph.D degree in Computer Science from University of Mysore in the year 2019. He is currently working as Assistant Professor in Department of Computer Science, Amrita School of Arts and Sciences, Mysuru Campus. He has published more than $30+$ research articles in reputed International journals and conferences and indexed in SCOPUS/SCI. His areas of research include machine learning, video processing, object detection, pattern classification and computer vision. He can be contacted at email: manohar@my.amrita.edu.

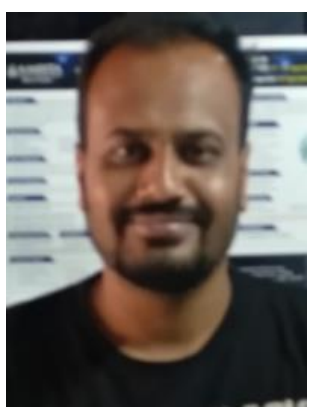

Hari Prasad M. (D) IS SC P is the final year student of MCA pursuing dissertation in the area of optical character recognition and deep learning at Amrita School of Arts and Science, Mysuru campus. His areas of research include document image processing and character recognition. He can be contacted at email: hariprasadm@my.amrita.edu.

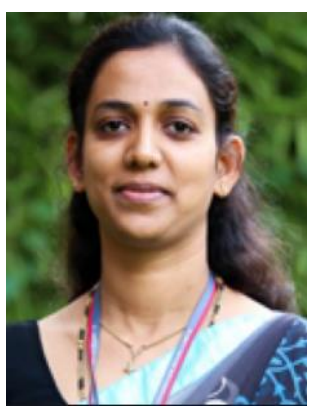

Pushpa B. R. (D) 8D SC P is pursuing her doctoral program in the area of computer vision and pattern recognition. She is also working as Assistant Professor in the Department of Computer Science. She has presented in various international conferences and also published more than $20+$ research articles in reputed international journals. Her areas of research include computer vision, object detection and recognition, deep learning and machine learning. She can be contacted at email: br_pushpa@my.amrita.edu. 